\title{
TDAH - diagnóstico e papel do professor
}

\author{
ADHD - diagnosis and teacher's role \\ TDAH - diagnóstico y rol del maestro
}

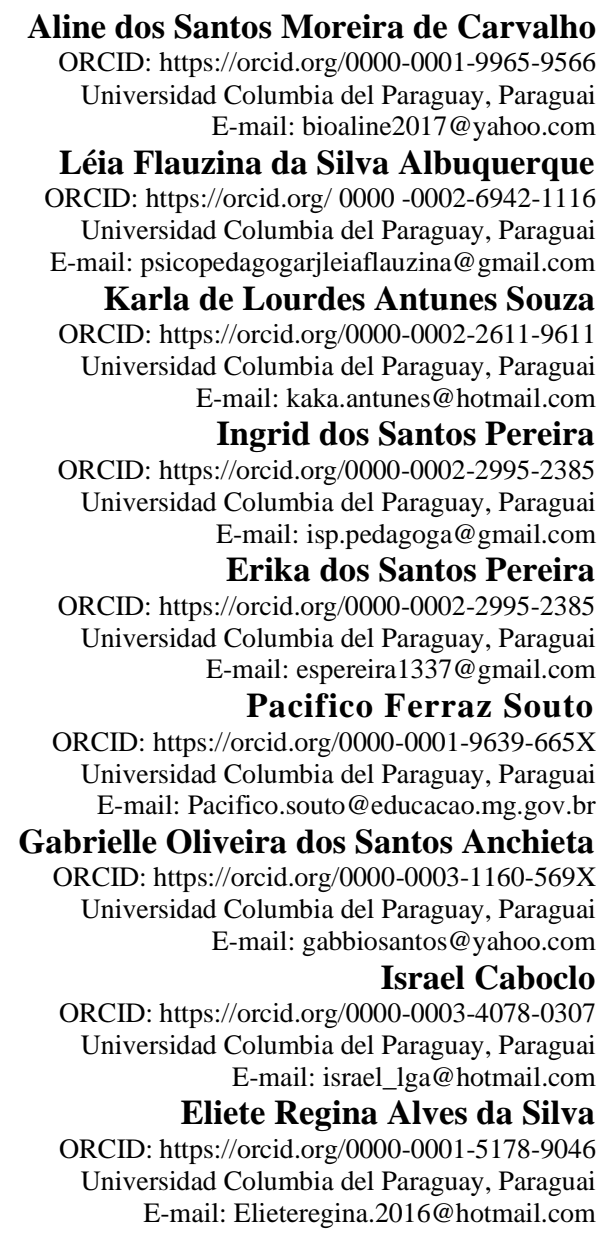

\begin{abstract}
Resumo
O Transtorno de Déficit de Atenção e Hiperatividade surgiu na literatura médica em meados do século XIX e passou por inúmeras mudanças de nomenclatura até o termo oficial atual, através do DSM-V. Sendo um transtorno neurobiológico de causas genéticas, o transtorno apresenta três sintomas principais que são: hiperatividade, desatenção e impulsividade e sua conceituação relaciona a psiquiatria, psicologia e a educação, pois a escola é o primeiro lugar onde os sintomas ficam evidenciados e podem ser melhor observados. As especificidades do diagnóstico e da avaliação diagnóstica fazem com que o professor exerça papel fundamental no processo, sendo responsável pelos apontamentos e observações acerca dos sintomas apresentados. O presente estudo por objetivo descrever o papel do professor no diagnóstico do TDAH. Para tal, foi utilizada como metodologia pesquisa de caráter qualitativo, em relação aos objetivos, exploratório e descritivo e no que concerne os procedimentos de pesquisa, o estudo caracterizou-se como bibliográfico. A pesquisa foi realizada em meio virtual, em bases de dados confiáveis como Scielo, com artigos científicos, livros em formato PDF, publicações e anais de congresso, com os descritores TDAH, diagnóstico, professor. Após a busca e análise, os resultados e discussão estão divididos em três tópicos, a saber: O transtorno, as especificidades no diagnóstico e o papel do professor no diagnóstico do TDAH. As conclusões e impressões da autora compreendem as considerações finais.
\end{abstract}

Palavras-chave: Diagnóstico; Professor; TDAH. 


\begin{abstract}
Attention Deficit Hyperactivity Disorder appeared in the medical literature in the mid-19th century and underwent numerous nomenclature changes until the current official term, through the DSM-V. Being a neurobiological disorder of genetic causes, the disorder has three main symptoms which are: hyperactivity, inattention and impulsivity and its conceptualization relates to psychiatry, psychology and education, as school is the first place where symptoms are evident and can be better observed. The specifics of diagnosis and diagnostic evaluation make the teacher play a fundamental role in the process, being responsible for the notes and observations about the symptoms presented. The present study aims to describe the role of the teacher in the diagnosis of ADHD. To this end, qualitative research was used as a methodology, in relation to the objectives, exploratory and descriptive, and with regard to research procedures, the study was characterized as bibliographic. The research was carried out in a virtual environment, in reliable databases such as Scielo, with scientific articles, books in PDF format, publications and conference proceedings, with the descriptors ADHD, diagnosis, professor. After the search and analysis, the results and discussion are divided into three topics, namely: The disorder, the specifics in the diagnosis and the role of the teacher in the diagnosis of ADHD. The author's conclusions and impressions comprise the final considerations.
\end{abstract}

Keywords: Diagnosis; Teacher; ADHD.

\title{
Resumen
}

El Trastorno por Déficit de Atención con Hiperactividad apareció en la literatura médica a mediados del siglo XIX y sufrió numerosos cambios de nomenclatura hasta el término oficial actual, a través del DSM-V. Al ser un trastorno neurobiológico de causas genéticas, el trastorno presenta tres síntomas principales que son: hiperactividad, desatención e impulsividad y su conceptualización se relaciona con la psiquiatría, la psicología y la educación, siendo la escuela el primer lugar donde los síntomas se manifiestan y se pueden observar mejor. Las especificidades del diagnóstico y la evaluación diagnóstica hacen que el docente juegue un papel fundamental en el proceso, siendo responsable de las notas y observaciones sobre los síntomas presentados. El presente estudio tiene como objetivo describir el papel del docente en el diagnóstico del TDAH. Para ello se utilizó como metodología la investigación cualitativa, en relación a los objetivos, exploratoria y descriptiva, y en cuanto a los procedimientos de investigación, el estudio se caracterizó como bibliográfico. La investigación se realizó en un ambiente virtual, en bases de datos confiables como Scielo, con artículos científicos, libros en formato PDF, publicaciones y actas de congresos, con los descriptores TDAH, diagnóstico, profesor. Después de la búsqueda y análisis, los resultados y la discusión se dividen en tres temas, a saber: El trastorno, las especificidades en el diagnóstico y el papel del docente en el diagnóstico del TDAH. Las conclusiones e impresiones del autor constituyen las consideraciones finales.

Palabras clave: Diagnóstico; Profesor; TDAH.

\section{Introdução}

O Transtorno de Déficit de Atenção e Hiperatividade surgiu na literatura médica por volta do século XIX e passou por diversas mudanças de nomenclatura no decorrer do tempo até o termo considerado oficial desde o DSM-IV, como hoje é conhecido, TDAH.

Considerado um transtorno neurobiológico de origem genética, o TDAH apresenta três principais sintomas que se desdobram em demais, que são: hiperatividade, desatenção e impulsividade.

Muitos autores convergem em defender que as descrições e conceitos do transtorno permeiam a psiquiatria, a psicologia e a educação, pois a escola é o lugar onde primeiramente os sintomas ficam evidenciados, uma vez que o TDAH geralmente surge em crianças na idade pré-escolar.

O TDAH apresenta algumas especificidades quanto ao diagnóstico detalhado ou avaliação diagnóstica, cabendo ao professor da criança, papel fundamental e incontestável nesse processo, sendo responsável pelos apontamentos e observações pertinentes àquele.

Diante da necessidade de destacar o papel do professor no diagnóstico do TDAH, o presente estudo por este seu objetivo: descrever o papel do professor no diagnóstico do TDAH.

Para tal, foi utilizada como metodologia pesquisa de caráter qualitativo pois tal abordagem oferece e possibilita uma visão para [...] o universo dos significados, dos motivos, das aspirações, das crenças, dos valores e das atitudes.” (Minayo et al., 2009, p. 21).

Em relação aos objetivos, é uma pesquisa exploratória e descritiva (GIL, 2008) pois busca descrever as nuances do diagnóstico do TDAH assim como o papel do professor durante o processo. 
No que concerne os procedimentos de pesquisa, o estudo caracterizou-se como bibliográfico que, segundo Gil (2008), é desenvolvida com base em material já elaborado e publicado como artigos científicos e livros. A pesquisa foi realizada em meio virtual, em bases de dados confiáveis como Scielo, com artigos científicos, livros em formato PDF, publicações e anais de congresso, com os descritores TDAH, diagnóstico, professor.

Após a busca e análise, os resultados e discussão estão divididos em três tópicos, a saber: $\mathrm{O}$ transtorno, as especificidades no diagnóstico e o papel do professor no diagnóstico do TDAH. As conclusões e impressões da autora compreendem as considerações finais.

\section{O Transtorno}

As primeiras informações e descrição de transtornos hipercinéticos surgiram na literatura médica em meados do século XIX, porém a nomenclatura tem mudado bastante com o decorrer do tempo. Um 1940 recebeu o nome de "lesão cerebral mínima" que, em 1962, passou para disfunção cerebral mínima (reconhecido que há disfunção e não lesão cerebral). Os sistemas classificatórios como CID e DSM apresentaram mesmo conceito, porém nomenclatura diferentes: no primeiro, transtornos hipercinéticos e no segundo transtorno de déficit de tenção e hiperatividade, utilizado largamente (Rohde et al., 2000).

Estudos realizados ao longo do tempo confirmam a média prevalente do transtorno, em crianças em idade pré-escolar, entre 3 a 8\%, considerando o impacto causado na sociedade, relevante, uma vez que tem alto custo financeiro, gera grandes estresses familiares, há prejuízo das atividades acadêmicas e principalmente problemas a níveis individuais como baixa autoestima (Rohde et al., 2000).

Barkley (2008, p. 17 apud Bonadio; Mori, 2013, p. 27) afirma que as crianças afetadas eram "descritas como “[...] limitadas em sua atenção, na regulação das atividades e da impulsividade, bem como outras características cognitivas, incluindo a memória".

O TDAH é um transtorno neurobiológico, de origem genética, que aparece na infância e geralmente acompanha o indivíduo até a fase adulta. É observado quando o mesmo apresenta comportamentos discrepantes com a idade e inteligência, o que gera prejuízo no aspecto de integração social (Shimizu; Miranda, 2012 apud Costa et al., 2020).

Os sintomas mais constatados são a hiperatividade e/ou a falta de atenção (gerando interferências no desempenho educacional, social ou ocupacional), seguido pela impulsividade (impaciência, problemas em esperar, interromper as pessoas falando antes que terminem). Podem apresentar também baixa tolerância a frustrações, fragilidade emocional, problemas com autoestima, entre outros (Oliveira, 2017 apud Costa et al., 2020).

A classificação do transtorno é realizada em três tipos diferentes: desatento - não enxerga os detalhes, comete erros por descuido, parece que não ouve, tem dificuldades em seguir instruções, é desorganizado, evita tarefas com muito esforço mental, esquece tarefas do cotidiano; hiperativo/ impulsivo - inquieto, mexe-se e remexe-se diversas vezes, não consegue ficar sentado, fala de4masiadamente; e o tipo combinado - quando apresenta características dos tipos anteriores conjuntas (Shimizu; Miranda, 2012; Gonçalves, 2018 apud Costa et al., 2020).

De acordo com Condemarín et al. (2006 apud Azevedo, 2015) existe variedades de definições teóricas, de acordo com os direcionamentos de estudos aos quais pertencem, trazendo certa complexidade para o diagnóstico e tratamento.

\section{As Especificidades no Diagnóstico}

Pereira afirma que o discurso acerca da descrição e conceituação do Transtorno de Déficit de Atenção e Hiperatividade (TDAH) é traçado mediante a contribuição da psiquiatria, psicologia e educação uma vez que, neste contexto, são analisadas questões comportamentais, a fim de desenvolver a legitimação do TDAH no campo científico e educacional (Pereira, 2009). 
Barkley (2008 apud Bonadio \& Mori, 2013) afirma que a correlação estabelecida entre sintomas físicos e a anatomia não favoreceu para que esclarecimentos fossem realizados, assim como Cypel (2007 apud Bonadio \& Mori, 2013) afirma que existe a preocupação em encontrar em bases orgânicas, sinais que poderiam comprovar o diagnóstico.

Diante da complexidade de provar o diagnóstico através de evidências anatômicas, houve algumas controvérsias, incluindo o aspecto da educação como compreendida no contexto do diagnóstico, o que levou a uma confusão de termos e maior dificuldade na compreensão do transtorno (Cypel, 2007 apud Bonadio \& Mori, 2013).

Silva (2003 apud Azevedo, 2015) afirma que o TDAH interfere diretamente na área cerebral que controla os impulsos e filtra os estímulos. Para a autora, as crianças com TDAH:

Possuem dificuldades muito específicas derivadas de seu também muito específico funcionamento cerebral, e que isso não deve ser confundido obrigatoriamente com tolice, má-educação ou dificuldades intelectuais. Só que, como é enorme o desconhecimento do problema, é exatamente isso o que acontece na grande maioria dos casos (Silva, 2003, p.59 apud Azevedo, 2015, p.22).

Para Rohde e Benczik (1999 apud Azevedo, 2015), não é necessário apresentar todos sos sintomas de uma vez, mas são sempre muitos que ocorrem juntamente e não todos.

Rohde et al. (2000) afirma que as crianças com TDAH são facilmente reconhecidas em ambientes como escola, clínica e casa. Acredita-se que o diagnóstico do TDAH deve ser feito contextualizando os sintomas com o histórico de vida de cada criança, e que alguns detalhes afirmam a presença do transtorno, como: a duração dos sintomas do transtorno (desde a vida préescolar), aparição dos sintomas após um desencadeante psicossocial, frequência e intensidade dos sintomas (para o diagnóstico é necessário que pelo menos seis dos sintomas que se desdobram dos três principais estejam presentes frequentemente na vida desses indivíduos), persistência dos sintomas em diversos lugares e ao longo do tempo, prejuízo clinicamente significativo para a vida da criança, entendimento do significado do sintoma.

Hallowell e Ratey (1999, p. 63 apud Azevedo, 2015, p. 22) observam ser necessário analisar cuidadosamente a história de cada criança, pois o TDAH baseia-se não somente nos sintomas como também no histórico individual de cada um e afirmam que ““'a ferramenta diagnóstica mais confiável é a história de cada caso, conforme extraída da criança, de seus pais e, o que é muito importante, dos relatórios dos professores".

Goldstein E Goldstein (1994, p. 41 apud Azevedo, 2015) afirmam que para a realização de um diagnóstico detalhado, faz-se necessário observar oito tipos de informações, das quais: histórico - o relato sobre a história e contexto familiar é extremamente importante como fonte de informação; inteligência -

Crianças com inteligência abaixo da média ficam provavelmente muito mais frustradas pelas exigências cada vez mais complexas impostas pela escola e pela vida. Assim, elas têm mais probabilidade de apresentar problemas de hiperatividade como resultado de frustração e não necessariamente decorrente de uma dificuldade temperamental. (Goldstein \& Goldstein, 1994, p. 42 apud Azevedo, 2015, p. 23).

Personalidade e desempenho emocional - é feita a observância do nível de confiança das crianças e seus níveis de satisfação; desempenho escolar - determinar habilidades escolares; amigos - facilidade ou dificuldade em fazer amigos é um determinante do desenvolvimento emocional; comportamento em casa -

A maneira como os pais integram com a criança não necessariamente é a causa do TDAH, mas é um fator que determina o nível de gravidade dos problemas que a criança hiperativa tem em casa". (Goldstein; Goldstein, 1994, p. 43 Azevedo, 2015, p. 23). 
Comportamento em sala de aula - percepções e observações do professor e consulta médica - diagnóstico clínico como parte da avaliação.

Condemarín et al. (2006 apud Azevedo, 2015) afirma que as crianças com TDAH constituem um grupo heterogêneo e amplo necessitando de condições especiais para a avaliação diagnóstica e alegam a necessidade de uma abordagem multidisciplinar que inclua especialistas de diversas áreas assim como os pais, responsáveis e os professores.

Nessa perspectiva, muitos autores concordam que a relação existente entre o discurso psiquiátrico e o educacional auxilia o diagnóstico, uma vez que a escola é o ambiente mais comum onde são observados os sintomas do transtorno.

\section{O Papel do Professor no Diagnóstico do TDAH}

Parker (2006 apud Azevedo, 2015) acredita que os professores são os primeiros e que com maior frequência observam problemas de comportamento, atenção, aprendizagem e emocionais.

Para Pereira (2009), quando o diagnóstico psiquiátrico do TDAH confere ao fracasso escolar, ser uma consequência do transtorno, esse funciona como um mecanismo de significação para a indisciplina e desatenção e passa a fazer uma ponte na relação estabelecida entre aluno, professor e família. E acrescenta que quando o professor se apropria desse diagnóstico, uma ação possível nesse sentido passa a ser estabelecida (desadaptação).

Cardoso (2009) afirma que, o entendimento por parte dos professores acerca do transtorno e de suas implicações trará benefícios para o diagnóstico multidisciplinar, alinhando parcerias entre a saúde e a educação, ou seja, conforme as situações específicas forem acontecendo, cabe aos professores buscarem maiores conhecimentos, capacitando-se para sinalizarem os sintomas evidentes e recorrentes, como a hiperatividade, desatenção e impulsividade manifestos em sala de aula.

Goldstein S. e Goldstein M. (2003, p. 79 apud Cardoso, 2009, p. 247) afirmam que:

Os professores da pré-escola podem e devem ser treinados a identificar crianças pré-escolares sob o risco não apenas de problemas de hiperatividade, mas também de sinais precoces de incapacidade de aprendizado e outros distúrbios psicológicos, como aqueles relacionados com a ansiedade e a depressão.

Nesse sentido, o professor atento, cabe a esse treinar o olhar para perceber os sintomas incontroláveis do TDAH, uma vez que o desenvolvimento cognitivo e psíquico desse indivíduo está sob sua responsabilidade (Cardoso, 2009).

O professor exerce papel fundamental no controle do comportamento de crianças TDAH, assim como no desenvolvimento de suas habilidades como ser humano. Portanto, deve estar em constante atualização como tal para contribuir com o diagnóstico, tratamento e inclusão dessas crianças (Santos; Franke, 2017 apud Costa et al., 2020).

Caliman (2006, p.102 apud Pereora, 2009, p. 7) afirma que na década de 1990, "o TDAH foi legitimado, popularizado, universalizado e tornou-se motivo de polêmica", uma vez que sua amplitude social no Ocidente trouxe uma significância por vezes estendida as crianças que possuem comportamentos desafiadores em ambiente escolar e institucional.

Uma questão a ser observada neste contexto é a de não generalização: nem toda criança hiperativa apresenta o TDAH, por vezes é uma questão de comportamento ativo normal para a idade (Garcia, 2001 apud Cardoso, 2009).

Muitos autores consideram incontestável a importância do professor para o diagnóstico do TDAH, porém observam que esse grupo de profissionais não é o responsável pelo diagnóstico, mas que esses devem compartilhar com os demais profissionais tanto as observações como alterações ou acontecimentos ocorridos em classe (Rief \& Heimburge, 2000 apud Cardoso, 2009).

Sendo assim, o professor é fundamental para a sinalização dos sintomas do TDAH presentes em alunos, observando seus alunos, suas reações mediante regras e ordens estabelecidas, como ele aprende, seu relacionamento com os demais, o seu comportamento e avaliar sua aprendizagem, conhecendo melhor o aluno e suas habilidades individuais (Cardoso, 2009). 
De acordo com a APA (1994, p. 84 apud Azevedo, 2015, p. 17), alguns itens referentes a alunos TDAH podem ser pinçados do discurso escolar: ": "frequentemente deixa a cadeira na sala de aula ou em outras situações nas quais ficar sentado é esperado", "frequentemente responde às perguntas antes de ouvi-las por inteiro" ou "frequentemente não segue a contento instruções ou não termina trabalhos, tarefas ou deveres no espaço de trabalho".

Portanto, a escola é o lugar onde o TDAH é percebido e fica evidente de ser observado. Sendo assim, os professores têm fundamental importância para a sinalização de sintomas e posterior diagnóstico do TDAH em crianças.

\section{Considerações Finais}

O TDAH é considerado um transtorno neurobiológico, com sintomatologia prevalente em tríade (hiperatividade, desatenção e impulsividade) que possui desdobramentos em demais sintomas.

As especificidades na realização do diagnóstico, que permeiam a psiquiatria, psicologia e a educação fazem com que o professor possua papel fundamental no diagnóstico assim como no tratamento e inclusão da criança portadora de TDAH.

A avaliação diagnóstica feita de forma minuciosa, conta com a contribuição das observações e apontamentos do professor sobre os alunos, o que implica em melhores informações para um diagnóstico preciso, assim como futuras intervenções terapêuticas.

Portanto, o professor é peça importante para o diagnóstico e incontestavelmente a pessoa que mais contribui para o desenvolvimento cognitivo da criança.

\section{Referências}

Amorim, C. (2017). O TDAH tem cura? HTTPS://dda deficitdeatencao.com.br/ artigos/tdah-tem-cura.html

Arruda, M. A. Levados da Breca - um guia sobre crianças e adolescentes com Transtorno do Déficit de Atenção e Hiperatividade (TDAH). Pdf. Ribeirão Preto. 2006. < https://www.estantevirtual.com.br/livros/marco-a-arruda/levados-da-breca/2178283709>.

Azevedo, R. M. D. $O$ TDAH na perspectiva da inclusão. Universidade Cândido Mendes, 37 f., Brasília, $2015 . \quad<$ https://www.avm.edu.br/docpdf/monografias_publicadas/posdistancia/50640.pdf>.

Barkley, R. A. (2020) Transtorno de déficit de atenção/hiperatividade - Manual para diagnóstico e tratamento. Pdf. (3a ed.). Artmed. https://grupoautentica.com.br/autentica/livros/tdah-transtorno-do-deficit-de-atencao-com-hiperatividade/1831>.

Bonadio, R. A. A., \& Mori, N. N. R. (2013) Transtorno de déficit de atenção/ hiperatividade: diagnóstico da prática pedagógica Eduem, 251 p.

Caliman, L. V. Notas sobre a história oficial do transtorno do déficit de atenção/hiperatividade TDAH. Psicologia Ciência, 30(1), 2010. < https://www.scielo.br/j/pcp/a/K7H6cvLr349XXPXWsmsWJQq/?lang=pt>.

Cardoso, D. M. P. O fazer pedagógico diante do transtorno de deficit de atenção e hiperatividade no contexto escolar. In: DÍAZ, F., et al., orgs. Educação inclusiva, deficiência e contexto social: questões contemporâneas EDUFBA, 245-254.

Costa et al., Mauritânia Lima de Oliveira. Perspectivas de alunos com TDAH na Educação Básica Pública Brasileira. Anais. CONEDU, Maceió, 2020. < https://editorarealize.com.br/editora/anais/conedu/2020/TRABALHO_EV140_MD1_SA10_ID5845_01092020111333.pdf.

Couto, T. S., Júnior, M. R. M. \& Gomes, C. R. (2010). Aspectos Neurobiológicos do Transtorno do Déficit de Atenção e Hiperatividade (TDAH): uma revisão. Revista Ciências e Cognição. http://cienciasecognicao.org/revista/index.php/cec/article/view/2021.

Cypel, S. (2003). A Criança com Déficit de Atenção e Hiperatividade: Atualização para pais, professores e profissionais de saúde. (2a ed.), Lemos Editorial.

Dsm-IV. (2003). Associação Americana de Psiquiatria. Manual diagnóstico e estatístico de transtornos mentais - DSM. (4a ed.), Tradução Claudia Dornelles. Porto Alegre: Artes Médicas.

Domingues, L. (2014). TDAH - Tratamento. blog.saude.gov. http://www.blog.saude.gov.br/34273-tdah-atinge-de-3-a-6-da-populacao-mundial-saiba-maissobre-o-transtorno.html.

Dupin, A. A. S. Q. \& Silva, M. O. (2020). Educação Especial e Legislação brasileira: revisão de literatura. Scientia Vitae. http://revistaifspsr.com/v10n297690.pdf.

Ferreira, R. R., \& Moscheta, M. dos S. A Multiplicidade do TDAH nas Diferentes Versões Produzidas pelas Ciências no Brasil. Psicologia Escolar e do Desenvolvimento, Psicologia: Teoria e Pesquisa, 35, < https://www.scielo.br/j/ptp/a/qwcxTtB4FJSvJMBBtQpqBNK/?lang=pt $>$.

Freire, A. C. C., \& Pondé, M. P. (2005). Estudo piloto da prevalência de TDAH entre crianças escolares na cidade de Salvador, Bahia, Brasil. Arquivos de Neuropsiquiatria, 63(2), 474-478. 
Research, Society and Development, v. 11, n. 2, e17711225724, 2022

(CC BY 4.0) | ISSN 2525-3409 | DOI: http://dx.doi.org/10.33448/rsd-v11i2.25724

Gil, A. C. (2008) Métodos e técnicas da pesquisa social. (6a ed.), Atlas.

Minayo, M. C. de S., et al. (2009) Pesquisa Social: teoria, método e criatividade. (28a ed.), Vozes.

Nunes. A. N. F. Publicada lei que assegura direitos a alunos com TDAH e outros transtornos de aprendizagem. Ferreiranunes - advocacia em direito educacional, $<$ http://ferreiranunesadvocacia.com.br/publicada-lei-que-assegura-direitos-alunos-com-tdah-e-outros-transtornos-de-aprendizagem/.

Pereira, C. de S. C. Conversas e controvérsias: uma análise da constituição do TDAH no cenário científico e educacional brasileiro. Pdf. Casa de Oswaldo Cruz, 176 f., < http://www.ppghcs.coc.fiocruz.br/images/teses/dissertacaoclaricedesa.pdf.

Rezende, E. Leis e direitos de alunos com TDAH: o que a legislação diz? Psicoedu - Psicologia e Educação, 2021. <https://www.psicoedu.com.br/2016/11/leitdah-

direitos.html\#: :text=N\%C3\%A3o\%20existe\%20legisla\%C3\%A7\%C3\%A3o\%20nacional\%20espec\%C3\%ADfica\%20que\%20ampare\%20alunos\%20diagnos ticados\%20com\%20TDAH.\&text=Embora\%20possamos\%20interpretar\%20que\%20esses,um\%20atendimento\%20diferenciado\%20destes\%20alunos.>.

Rohde L. A., et al. (2000) Transtorno de déficit de atenção/hiperatividade. Brazilian Journal Psychiatry, 22(2), https://www.scielo.br/j/rbp/a/zsRj5Y4Ddgd4Bd95xBksFmc/?lang=pt. 